\section{DESENVOLVIMENTO E REPRODUTIBILIDADE DO INSTRUMENTO DE AVALIAÇÃO DA PROMOÇÃO DA SAÚDE NA UNIVERSIDADE - IAPSU}

\author{
Development and reproducibility of the instrument for \\ assessment of health promotion in university - IAPSU
}
Desarrollo y reproductibilidad del instrumento de evaluación de la promoción de la salud de la universidad - IAPSU

\section{RESUMO}

Objetivo: Apresentar o Instrumento de Avaliação da Promoção da Saúde na Universidade (IAPSU) e seu processo de avaliação de reprodutibilidade. Métodos: Estudo transversal realizado entre maio e julho de 2014 com 50 acadêmicos de uma universidade de FortalezaCE, o qual desenvolveu o IAPSU a partir da análise de documentos governamentais e de uma revisão sistemática da literatura acerca de uma universidade potencialmente saudável. O instrumento possui 41 questões, divididas em cinco domínios: atividade física, alimentação, fatores ambientais, fatores psicossociais e consumo de álcool e drogas, e práticas integrativas e complementares. Para avaliação da reprodutibilidade interobservador, os acadêmicos responderam duas vezes ao questionário, aplicado por examinadores distintos; para a avaliação intraobservador, outra aplicação do instrumento ocorreu sete dias depois. Resultados: Participaram do estudo 40 alunos do curso de Enfermagem e 10 do curso de Fisioterapia, com idade média de $25 \pm 5,4$ anos, sendo $88 \%$ do sexo feminino e predomínio da raça branca. Na análise da reprodutibilidade, foram observados fortes coeficientes de correlação intraclasse, intraexaminador e interexaminador, acima de 0,8 em todos os domínios estudados. Conclusão: Conclui-se que o IAPSU é um instrumento reprodutível e confiável para avaliação da promoção da saúde no âmbito universitário.

Descritores: Promoção da Saúde; Universidades; Avaliação em Saúde.

\section{ABSTRACT}

Objective: To present the instrument for assessment of health promotion in university (IAPSU) and its reproducibility assessment process. Methods: Cross-sectional study performed between May and July 2014 with 50 students from one university of Fortaleza, $C E$, which developed the IAPSU through the analysis of governmental documents and a systematic literature review addressing a potentially healthy university. The instrument has 41 questions, divided into five domains: physical activity, feeding, environmental factors, psychosocial factors and alcohol and drugs consumption, complementary and integrative practices. To evaluate the inter-rater reproducibility, the students answered the IAPSU twice, applied by two different examiners; to evaluate the intra-rater reliability, another application of the instrument was performed after seven days. Results: The study comprised 40 students of the Nursing course and 10 of the Physiotherapy course, with mean age of $25 \pm 5.4$ years; $88 \%$ were female and Caucasians were predominant. In the reproducibility assessment, strong intraclass, intra-rater and inter-rater correlation coefficients, over 0.8 , were observed in all the assessed domains. Conclusion: The IAPSU is a reproducible and reliable instrument to evaluate health promotion in the university environment.

Descriptors: Health Promotion; Universities; Health Evaluation.
Artigo Original

Ana Maria Fontenelle Catrib(1) Natália Bitar da Cunha Olegario $^{(2)}$

Daniela Gardano Bucharles Mont'Alverne ${ }^{(3)}$

Guilherme Pinheiro Ferreira da Silva ${ }^{(1)}$

Ana Paula Vasconcellos Abdon ${ }^{(1)}$ Juliana Guimarães e Silva ${ }^{(1)}$ Nelson Filice de Barros $^{(4)}$

1) Universidade de Fortaleza - UNIFOR Fortaleza (CE) - Brasil.

2) Faculdade de Tecnologia Intensiva FATECI - Fortaleza (CE) - Brasil

3) Universidade Federal do Ceará - UFC Fortaleza (CE) - Brasil.

4) Universidade Estadual de Campinas UNICAMP - Campinas (SP) - Brasil.

Recebido em: 01/09/2015 Revisado em: 20/09/2015 Aceito em: 30/09/2015 


\section{RESUMEN}

Objetivo: Presentar el Instrumento de Evaluación de la Promoción de la Salud de la Universidad (IAPSU) y su proceso de evaluación de reproductibilidad. Métodos: Estudio transversal realizado entre mayo y julio de 2014 con 50 académicos de una universidad de Fortaleza-CE, el cual ha desarrollado el IAPSU a partir del análisis de documentos del gobierno y de una revisión sistemática de la literatura sobre una universidad saludable en potencia. El instrumento tiene 41 cuestiones divididas en cinco dominios: actividad física, alimentación, factores ambientales, factores psicosociales y el consumo del alcohol y drogas $y$ practicas integrativas y complementarias. Para la evaluación de la reproductibilidad interobservador los académicos contestaron dos veces al cuestionario que fue aplicado por examinadores distintos; otra aplicación del instrumento se dio siete días después para la evaluación intraobservador. Resultados: Participaron del estudio 40 alumnos del curso de Enfermería y 10 del Curso de Fisioterapia con edad media de $25 \pm 5$,4 años, siendo el $88 \%$ del sexo femenino y predominio de la raza blanca. En el análisis de la reproductibilidad fueron observados fuertes coeficientes de correlación intraclase, intraexaminador y interexaminador por encima de 0,8 en todos los dominios estudiados. Conclusión: Se concluye que el IAPSU es un instrumento reproductible y confiable para la evaluación de la promoción de la salud en el ámbito de la universidad.

Descriptores: Promoción de la Salud; Universidades; Evaluación en Salud.

\section{INTRODUÇÃO}

A promoção da saúde (PS) é uma estratégia de articulação transversal na qual se confere visibilidade aos fatores que colocam a saúde da população em risco e às diferenças de necessidades, territórios e culturas, tendo em vista a criação de mecanismos que reduzam as situações de vulnerabilidade, defendam a equidade e incorporem a participação e o controle social na gestão das políticas públicas. Nesse sentido, a elaboração da Política Nacional de Promoção da Saúde (PNPS) ${ }^{(1)}$ no ano de 2001 é a base para o processo de construção e implementação de um novo modo de organizar, planejar, realizar, analisar e avaliar o trabalho em saúde.

Para a implementação da PNPS no Brasil, um dos pontos estratégicos foi o estímulo à criação de Escolas Promotoras de Saúde (EPS), com foco em ações de alimentação saudável, práticas corporais/atividades físicas e ambiente livre de tabagismo ${ }^{(2)}$. Os espaços educacionais constituem-se em ambientes ideais para o desenvolvimento de comportamentos promotores de saúde e adoção de estilos de vida saudáveis. Sobretudo porque muitas das condutas pouco saudáveis e que geram agravos à saúde são construídas na infância e na juventude, incorporando-se de tal forma que modificá-las posteriormente é difícil ${ }^{(3-5)}$.

As universidades são instituições onde as pessoas aprendem, trabalham, socializam e usufruem do seu tempo de lazer, além de utilizarem serviços oferecidos. Assim, têm significativo potencial para proteger a saúde e promover o bem-estar de estudantes, funcionários e comunidade por meio de políticas e práticas de promoção de saúde, pois formam pessoas que são ou serão profissionais e formuladores de políticas com potencial de modificar condições que afetam a qualidade de vida de pessoas ${ }^{(6)}$.

Embora o movimento de promoção tenha sido iniciado há mais de quatro décadas e produzido um grande número de documentos nacionais e internacionais, não tem sido dado destaque a estudos que possibilitem identificar a universidade como espaço propício para o desenvolvimento de ambientes saudáveis, assim como para a produção de instrumentos reprodutíveis que explorem a realidade dos ambientes acadêmicos. Considerando que os espaços universitários são propícios para se desenvolver ações de promoção de saúde e não estão contemplados nas políticas que visam à construção de ambientes educacionais saudáveis no Brasil, é de fundamental importância a criação de instrumentos que objetivem avaliar as ações de promoção da saúde no âmbito universitário.

O objetivo do estudo foi apresentar o Instrumento de Avaliação da Promoção da Saúde na Universidade (IAPSU) e seu processo de avaliação de reprodutibilidade.

\section{MÉTODOS}

Realizou-se um estudo de caráter transversal, entre maio e julho de 2014, com acadêmicos de uma universidade privada situada em Fortaleza-CE.

Utilizaram-se como critérios de inclusão alunos regularmente matriculados nos cursos de Enfermagem e Fisioterapia, com idade superior a 18 anos, independentemente do sexo, sendo excluídos alunos que não realizaram o preenchimento completo solicitado durante a aplicação do instrumento e/ou não finalizaram o protocolo de reprodutibilidade proposto no estudo.

A amostra do tipo não probabilística e por conveniência foi composta por 50 acadêmicos, corroborando com os parâmetros descritos para realização de estudos de validação e reprodutibilidade de questionários ${ }^{(7)}$.

O Instrumento de Avaliação da Promoção da Saúde na Universidade - IAPSU (Figura 1) foi desenvolvido por pesquisadores da Universidade de Fortaleza e Universidade Estadual de Campinas, a partir da análise criteriosa de documentos governamentais e de uma revisão sistemática da literatura sobre a universidade potencialmente 
saudável ${ }^{(8)}$. Assim, elaborou-se um instrumento de coleta de dados contendo um módulo de identificação e 50 questões sobre a promoção da saúde na universidade, envolvendo: condicionantes do trabalho com promoção da saúde; trabalho docente com a promoção da saúde; e ações de gestão para a promoção da saúde na universidade.

Em um primeiro momento, na aplicação do instrumento piloto, participaram 20 acadêmicos, instruídos a registrar qualquer observação que porventura comprometesse a interpretação e o preenchimento do instrumento no alcance de identificar as dimensões que convergem para a existência de uma universidade saudável. Posteriormente, realizaramse os ajustes necessários, a partir das queixas apontadas pelos respondentes quanto a dificuldades semânticas e extensão do questionário, de forma que foram mantidos os indicadores sociais e demográficos (dados de identificação com 12 itens) e reduzidas nove questões do instrumento piloto, o que resultou em 41 quesitos, divididos em cinco domínios: atividade física (9 questões), alimentação (6 questões), fatores ambientais (5 questões), fatores psicossociais e consumo de álcool e drogas (18 questões), e práticas integrativas e complementares (3 questões).

Os domínios foram compostos pelos seguintes aspectos: a) atividade física: participação em atividades promovidas pela universidade; participação em atividades recreativas; espaço destinado à atividade física, influência da prática da atividade física na saúde e bem-estar; prática de atividade física ou recreativa por membros da família; b) alimentação: oferta de alimentos saudáveis por restaurantes ou lanchonetes; conceito de alimentação saudável; oferta de ambiente confortável para alimentação; participação em atividades sobre alimentação saudável na universidade; c) fatores ambientais: abordagens sobre uso consciente de água e energia; limpeza e funcionamento das instalações sanitárias; presença de lixeiras com tampas; iluminação, ventilção, acústica e mobiliário das salas de aula; sensação de segurança na universidade; d) fatores psicossociais e consumo de álcool e drogas: participação em atividades que envolvem fatores psicossociais; participação em atividades acadêmicas; relacionamento entre alunos, professores e coordenação do curso; situações de violência na universidade; conceito de universidade saudável; consumo de álcool, tabaco ou alguma droga ilícita na universidade; consumo de álcool e drogas por familiares; participação em atividades de prevenção de consumo de álcool e drogas na universidade; e) conhecimento e uso de práticas integrativas e complementares.

Para avaliação da reprodutibilidade interobservador do IAPSU, os alunos responderam duas vezes ao questionário, aplicado por dois examinadores distintos, separados por um intervalo de 30 minutos. A reprodutibilidade intraobservador foi realizada sete dias após a primeira, sendo o IAPSU aplicado novamente aos mesmos participantes por somente um dos examinadores.

Para realização dos testes estatísticos de reprodutibilidade, atribuiu-se a cada domínio um escore, a partir da soma das pontuações das questões, obtidas durante a aplicação do instrumento. Os itens das questões representavam a soma ou subtração de um ponto, de acordo com seu reflexo positivo ou negativo na construção de um ambiente saudável. As somatórias máximas de escores referentes a cada domínio foram: 41 (atividade fisica), 18 (alimentação), 12 (fatores ambientais), 63 (fatores psicossociais e consumo de álcool e drogas) e 46 (práticas integrativas e complementares). Para melhor descrição dos resultados do IAPSU, os escores obtidos foram transformados em percentuais.

Analisaram-se estatisticamente os dados obtidos por meio do programa SPSS, versão 17.0. Para análise das variáveis descritivas, foram utilizados média, desvio padrão e percentual. Utilizou-se para a análise da reprodutibilidade da aplicação do IAPSU o Coeficiente de Correlação Intraclasse (CCI). Adotou-se nível de significância de 5\%.

O presente estudo respeitou os preceitos éticos que regem as pesquisas em seres humanos, conforme as normas da Resolução $n^{\circ}$ 466/12 do Conselho Nacional de Saúde ${ }^{(9)}$, sendo aprovado pelo Comitê de Ética em Pesquisa da Universidade de Fortaleza, com o parecer de número 054/2010. Todos os participantes assinaram o termo de consentimento livre e esclarecido.

\section{RESULTADOS}

Incluíram-se no estudo 65 alunos, sendo 15 excluídos por não completarem o protocolo proposto na pesquisa, sendo 40 do curso de Enfermagem e 10 do curso de Fisioterapia, com idade média de $25 \pm 5,4$ anos, predomínio do sexo feminino $(88 \%, \mathrm{n}=44)$ e da raça branca $(52 \%$, $\mathrm{n}=26$ ) (Tabela I). 
Tabela I - Características sociodemográficas dos acadêmicos participantes do estudo. Fortaleza-CE, 2014.

\begin{tabular}{|c|c|}
\hline Características sociodemográficas & $\mathbf{n} / \%$ \\
\hline Idade (anos) & $25 \pm 5,4$ \\
\hline \multicolumn{2}{|l|}{ Curso } \\
\hline Enfermagem (n/\%) & $40 / 80 \%$ \\
\hline Fisioterapia (n/\%) & $10 / 20 \%$ \\
\hline \multicolumn{2}{|l|}{ Sexo } \\
\hline Masculino (n/\%) & $6 / 12 \%$ \\
\hline Feminino $(\mathrm{n} / \%)$ & $44 / 88 \%$ \\
\hline \multicolumn{2}{|l|}{ Raça } \\
\hline $\operatorname{Branco}(\mathrm{n} / \%)$ & $26 / 52 \%$ \\
\hline Mulato/Pardo (n/\%) & $17 / 34 \%$ \\
\hline Amarelo (n/\%) & $6 / 12 \%$ \\
\hline Negro (n/\%) & $1 / 2 \%$ \\
\hline \multicolumn{2}{|l|}{ Religião } \\
\hline Católica (n/\%) & $41 / 82 \%$ \\
\hline Protestante $(\mathrm{n} / \%)$ & $8 / 16 \%$ \\
\hline Islâmica (n/\%) & $1 / 2 \%$ \\
\hline \multicolumn{2}{|l|}{ Faixa de renda familiar } \\
\hline $1 \mathrm{SM}(\mathrm{n} / \%)$ & $2 / 4 \%$ \\
\hline 2-5 SM (n/\%) & $22 / 44 \%$ \\
\hline 5-8 SM (n/\%) & $4 / 8 \%$ \\
\hline 8-11 SM (n/\%) & $3 / 6 \%$ \\
\hline 11-14 SM (n/\%) & $4 / 8 \%$ \\
\hline 14-17 SM (n/\%) & $2 / 4 \%$ \\
\hline 17-20 SM (n/\%) & $2 / 4 \%$ \\
\hline$>20 \mathrm{SM}(\mathrm{n} / \%)$ & $3 / 6 \%$ \\
\hline Não sabe (n/\%) & $8 / 16 \%$ \\
\hline \multicolumn{2}{|l|}{ Grau de escolaridade do pai } \\
\hline Ensino fundamental incompleto (n/\%) & $6 / 12 \%$ \\
\hline Ensino fundamental completo (n/\%) & $3 / 6 \%$ \\
\hline Ensino médio incompleto (n/\%) & $18 / 36 \%$ \\
\hline Ensino médio completo (n/\%) & $2 / 4 \%$ \\
\hline Ensino superior completo (n/\%) & $18 / 36 \%$ \\
\hline Ensino superior incompleto (n/\%) & $3 / 6 \%$ \\
\hline Não alfabetizado & $2 / 4 \%$ \\
\hline \multicolumn{2}{|l|}{ Grau de escolaridade da mãe } \\
\hline Ensino fundamental incompleto (n/\%) & $6 / 12 \%$ \\
\hline Ensino fundamental completo (n/\%) & $2 / 4 \%$ \\
\hline Ensino médio incompleto (n/\%) & $1 / 2 \%$ \\
\hline Ensino médio completo (n/\%) & $17 / 34 \%$ \\
\hline Ensino superior completo (n/\%) & $20 / 40 \%$ \\
\hline Ensino superior incompleto (n/\%) & $4 / 8 \%$ \\
\hline
\end{tabular}

$\mathrm{n}=$ número de indivíduos; $\%$ = porcentagem; > = maior que; $\mathrm{SM}=$ salário mínimo.

$\mathrm{Na}$ análise da reprodutibilidade dos instrumentos intraexaminador e interexaminador, observaram-se fortes coeficientes de correlação intraclasse, acima de 0,8 , em todos os domínios estudados (Tabela II). 
Tabela II - Análise da reprodutibilidade intraexaminador e interexaminador do Instrumento de Avaliação da Promoção da Saúde na Universidade (IAPSU), por meio do Coeficiente de Correlação Intraclasse. Fortaleza-CE, 2014.

\begin{tabular}{lcc}
\hline Domínios & CCI - Intraexaminador & CCI - Interexaminador \\
\hline Atividade Física & 0,89 & 0,90 \\
Alimentação & 0,81 & 0,91 \\
Fatores ambientais & 0,85 & 0,84 \\
Fatores psicossociais/CAD & 0,86 & 0,84 \\
Práticas Integrativas e Complementares & 0,92 & 0,86 \\
\hline
\end{tabular}

$\mathrm{CCI}=$ Coeficiente de Correlação Intraclasse; $\mathrm{CAD}=$ consumo de álcool e drogas.

$\mathrm{Na}$ comparação da pontuação total obtida entre a aplicação e a reaplicação do IAPSU por um mesmo observador, não se identificaram diferenças significativas, assim como quando comparadas as pontuações entre os dois examinadores do estudo ( $\mathrm{p}>0,05)$ (Tabela III).
Houve diferença estatística significante entre o tempo médio de aplicação do instrumento nos dois primeiros momentos de avaliação, sendo o tempo médio de aplicação do IAPSU no examinador 1 de $11,3 \pm 2,5$ minutos, e no examinador 2, de $7,2 \pm 2,1$ minutos $(\mathrm{p}<0,001)$.

Tabela III - Pontuação total obtida nas aplicações do Instrumento de Avaliação da Promoção da Saúde na Universidade (IAPSU) em acadêmicos. Fortaleza-CE, 2014.

\begin{tabular}{lccc}
\hline Domínios & Examinador 1 & Examinador 2 & Examinador 1.2 \\
\hline Atividade física (\%) & $39,8 \pm 5,5$ & $40,6 \pm 5,5$ & $40,7 \pm 5,2$ \\
Alimentação (\%) & $37,4 \pm 12,9$ & $35,9 \pm 13,3$ & $37,2 \pm 11,8$ \\
Fatores ambientais (\%) & $59,1 \pm 13$ & $59,7 \pm 14,2$ & $60,5 \pm 15,2$ \\
Fatores psicossociais/CAD (\%) & $51,1 \pm 12,1$ & $51,8 \pm 10,6$ & $51,4 \pm 9,9$ \\
PIC (\%) & $29,6 \pm 12,7$ & $27,9 \pm 11,6$ & $27,9 \pm 11,1$ \\
\hline
\end{tabular}

$\%=$ percentual; $\pm=$ desvio padrão; CAD = consumo de álcool e drogas; PIC = práticas integrativas e complementares; IAPSU = Instrumento de Avaliação da Promoção de Saúde na Universidade; Examinador 1.2 = reaplicação por um mesmo examinador.

\section{DISCUSSÃO}

No presente estudo, foi construído o Instrumento de Avaliação da Promoção da Saúde na Universidade (IAPSU), considerado reprodutível, que privilegiou a identificação das características do ambiente educacional universitário que interferem na saúde presente e futura da comunidade acadêmica, oferecendo, portanto, informações essenciais para a elaboração e execução de estratégias promotoras de saúde.

Destaca-se a escassez dessa temática na literatura internacional e no campo da saúde coletiva e da pesquisa em promoção da saúde no Brasil, nos quais não existem instrumentos similares ao IAPSU. Sua originalidade se deve à característica de reprodutibilidade para analisar ambientes universitários e, possivelmente, sua adaptação pode ser considerada para a pesquisa em outros campos do conhecimento, uma vez que instrumentos validados ou construídos na língua portuguesa avaliam apenas aspectos isolados da saúde.

A produção de um instrumento que possibilitasse a avaliação da promoção da saúde nos espaços educacionais do ensino superior foi realizada anteriormente ${ }^{(10)}$ por meio de um questionário autoaplicável para investigar comportamentos de saúde e características de estilo de vida. Foram incluídas informações sociodemográficas, nutricionais, de atividade física, sono reparador, tabagismo, uso de substâncias ilícitas, frequência de consumo excessivo de álcool e problemas com a bebida. Um estudo jordaniano utilizou outro instrumento autoaplicável para coleta de dados, incluindo aspectos sociodemográficos, peso, altura, relacionamento com a família e amigos, e semestre acadêmico ${ }^{(11)}$.

O questionário Health Promotion Life-style Profile- II (HPLP-II), disponibilizado em árabe e inglês, tem como objetivo a avaliação de comportamentos promotores de saúde e estilo de vida, composto por 52 itens e seis subescalas: responsabilidade com a saúde, atividade física, nutrição, crescimento espiritual, relações interpessoais e gestão de estresse $\mathrm{e}^{(12)}$.

Os questionários citados apresentam evidentes semelhanças com o instrumento proposto no presente estudo, pois objetivam, em sua maioria, investigar aspectos que envolvem a construção de ambientes 
propícios a comportamentos saudáveis e à incorporação destes no estilo de vida dos jovens. Outros instrumentos elaborados ou validados para a língua portuguesa propõem a avaliação de aspectos relacionados à saúde, porém, de forma isolada, como: o Questionário Internacional de Atividade Física (IPAQ), originalmente desenvolvido com a finalidade de estimar o nível de prática habitual de atividade física de populações de diferentes países ${ }^{(13)}$, e o Questionário Semiquantitativo de Frequência Alimentar (QSFA), elaborado para estimar o consumo alimentar de adolescentes $^{(14)}$.

No que se refere ao processo de avaliação da reprodutibilidade de questionários, alguns autores não citam a reprodutibilidade interexaminador ${ }^{(15,16)}$, procedimento efetuado neste estudo. Utilizou-se o protocolo de estudo anterior $^{(17)}$ para a avaliação da reprodutibilidade do questionário proposto. Por reprodutibilidade, entende-se uma comparação dos resultados obtidos por mais de um observador ou em mais de uma ocasião em uma mesma amostra de indivíduos ${ }^{(18)}$.

A importância da produção de instrumentos reprodutíveis que informem a realidade dos ambientes acadêmicos é permitir a construção de ações que promovam a saúde. Diferentes estudos permitem inferir que instituições universitárias deveriam promover ambientes saudáveis, uma vez que pesquisadores do Reino Unido, em 2011(10), concluíram que em sete universidades, na Inglaterra, País de Gales e Irlanda do Norte, apenas uma minoria dos estudantes exibiu práticas positivas de saúde, segundo recomendações internacionais no que se refere ao consumo de frutas e hortaliças e à prática de atividade física. $\mathrm{O}$ estudo evidenciou, ainda, que grande parte dos alunos estava envolvida em comportamentos de risco, como consumo excessivo de álcool e uso de drogas ilícitas.

Na Jordânia, pesquisa realizada com 340 acadêmicos de uma universidade pública mostrou que os alunos desenvolvem principalmente práticas espirituais, em detrimento de atividades físicas ${ }^{(11)}$. Outras investigações apontam que a saúde é um fator importante para o desempenho acadêmico, de forma que promover saúde e bem-estar significa, também, promover a aprendizagem efetiva $^{(18-22)}$.

Os programas de saúde escolar geram impacto positivo no desempenho acadêmico em instituições de ensino superior ${ }^{(21)}$, havendo associações positivas entre os parâmetros de saúde, como a prática de atividade física no ambiente educacional, e os resultados acadêmicos ${ }^{(22)}$.

As universidades possuem potencial para contribuir com a saúde em três áreas distintas: criação de ambientes de trabalho, aprendizagem e vivências saudáveis para estudantes e funcionários; ampliação da importância da saúde, promoção da saúde e da saúde pública no ensino e na pesquisa; e desenvolvimento de alianças e parcerias para a promoção da saúde e atuação comunitária ${ }^{(6)}$.

Considerando que estudantes universitários constituemse futuros tomadores de decisões nas organizações, comunidades e países, e que os anos de vida acadêmica são um período no qual os alunos cada vez mais fazem escolhas sobre seu estilo de vida e práticas de saúde ${ }^{(22)}$, esse ambiente deveria dar destaque à promoção da saúde e à construção de ambientes educacionais potencialmente saudáveis.

$\mathrm{Na}$ atual pesquisa, observou-se na aplicação do IAPSU a diferença estatística entre o tempo médio de aplicação do instrumento nos dois primeiros momentos de avaliação. Acredita-se que essa diferença seja resultado do efeito aprendizagem na reaplicação do instrumento, já conhecido pelo participante.

Na literatura ${ }^{(23)}$, preconiza-se que um valor de coeficiente de correlação intraclasse de 0,75 seja o mínimo aceitável para demonstrar a fidedignidade de um instrumento. A razão é que se esse valor for zero, há $50 \%$ de chance de ocorrer o inverso. Se o valor for 0,5 , o percentual cai para $37 \%$; 0,8 resulta em $20 \%$ de chance do contrário; 0,95 faz cair para $2,2 \%$. De modo geral, se o valor estiver acima de 0,6 , o resultado é aceito como um bom valor de correlação. No atual estudo, os valores de coeficiente de correlação intraclasse do IAPSU foram maiores que $0,8 \mathrm{em}$ todos os domínios, demonstrando forte correlação intraexaminador e interexaminador.

A aplicação do IAPSU possibilita o conhecimento do perfil de saúde dos universitários e contribui para a identificação das variáveis que interferem de forma positiva ou negativa no bem-estar e estilo de vida dos acadêmicos. Além disso, subsidia a criação e implantação de políticas educacionais com perspectiva crítica, orientadas para o processo de substituição de crenças, valores e hábitos que compõem os ciclos viciosos, a fim de constituir ciclos virtuosos. No entanto, há limitações no que se refere à falta de possibilidade de validação do instrumento através de tratamento estatístico, posto que não há outros questionários publicados em língua portuguesa com essa proposta de investigação. Sugere-se a sua adaptação para outras línguas, com o objetivo de viabilizar estudos em promoção da saúde no âmbito universitário em outros países, por ser uma alternativa viável para a realização de estudos sobre a temática.

É importante a realização de outros estudos que objetivem conhecer o ambiente acadêmico e possibilitem reconhecer seu potencial para determinar a mudança do estilo de vida e a aquisição de hábitos saudáveis. $\mathrm{O}$ movimento de promoção da saúde necessita aprofundar a discussão sobre a universidade como espaço promotor 
de saúde e desenvolver instrumentos que possibilitem a realização de mais pesquisas na comunidade acadêmica, com o fim de conhecer suas fragilidades, potencialidades e experiências exitosas, para embasar estratégias e ações de saúde no campo da educação.

\section{CONCLUSÃO}

Conclui-se que o IAPSU é um instrumento reprodutível e confiável para avaliação da promoção da saúde no âmbito universitário.

\section{REFERÊNCIAS}

1. Ministério da Saúde (BR), Secretaria de Gestão Estratégica e Participativa. A construção do SUS: histórias da Reforma Sanitária e do Processo Participativo. Brasília: Ministério da Saúde; 2006.

2. Catrib AMF, Gonçalves FD. Diseño evaluativo orientado al programa escuelas saludables para el aprendizaje de JUNAEB desarrollado entre losaños 2005-2007. Santiago: JUANEB; 2009.

3. Pires CGS, Mussi FC. Crenças em saúde para o controle da hipertensão arterial. Ciênc Saúde Coletiva. 2008;13(Supl 2):2257-67.

4. Gonçalves FD, Catrib AMF, Vieira NFC, Vieira LJES. A promoção da saúde na educação infantil. Interface Comun Saúde Educ. 2008;12(24):181-92.

5. Santos, ZMSA, Caetano JÁ, Moreira FGA. Atuação dos pais na prevenção da hipertensão arterial: uma tecnologia educativa em saúde. Ciênc Saúde Coletiva 2011;16(11):4385-94.

6. Mello ALSF; Moysés ST, Moysés SJ. A universidade promotora de saúde e as mudanças na formação profissional. Interface Comun Saúde Educ. 2010;14(34):683-92.

7. Cade J, Thompson R, Burley V, Warm D. Development, validation and utilisation of food-frequency questionnaires - a review. Public Health Nutr. 2001;5(4):567-87.

8. Catrib AMF, Silva RM. Promoção de saúde na adolescência e concepções de cuidados. Fortaleza: UECE; 2014.

9. Conselho Nacional de Saúde (BR). Resolução no 466, de 12 de dezembro de 2012. Aprova diretrizes e normas regulamentadoras de pesquisa envolvendo seres humanos. Diário Oficial da União; 13 jul 2013; Seção 1.
10. El Ansari W, Stock C, John J, Deeny P, Phillips C, Snelgrove $\mathrm{S}$, et al. Health promoting behaviours and lifestyle characteristics of students at seven universities in the UK. Cent Eur J Public Health: 2011;19(4):197204.

11. Al-Khawaldeh OA. Health promoting lifestyles of Jordanian university students. Int J Nurs Stud. 2014;3(1):27-31.

12. Walker SN, Sechrist KR, Pender NJ. The healthpromoting lifestyle profile: development and psychometric characteristics. Nurs Res. 1987;36(2):7681.

13. Guedes DP, Lopes CC, Guedes JERP. Reprodutibilidade e validade do Questionário Internacional de Atividade Física em adolescentes. Rev Bras Med Esporte. 2005;11(2):151-64.

14. Araújo MC, Ferreira DM, Pereira RA. Reprodutibilidade de questionário semiquantitativo de freqüência alimentar elaborado para adolescentes da Região Metropolitana do Rio de Janeiro, Brasil. Cad Saúde Pública. 2008;24(12):2775-86.

15. Novello AC, Degraw C, Kleinman, D. Healthy children ready to learn: an essential collaboration between health and education. Public Health Rep. 1992,107(1):3-15.

16. Tsouros AD, Dowding G, Thompson J, Dooris M. Health promoting universities- concept, experience and framework for action. Denmark: World Health Organization; 1998.

17. Silva GPF, Morano MTAP, Viana CMS, Magalhães CBA, Pereira EDB. Validação do Teste de Avaliação da DPOC em português para uso no Brasil. J Bras Pneumol. 2013;39(4):402-8.

18. O'Donnell T, Gray G. The health promoting college. London: Health Education Authority; 1993.

19. Murray NG, Low BJ, Hollis C, Cross AW, Davis SM. Coordinated School Health Programs and Academic Achievement: a systematic review of the literature. J School Health. 2007;77(9):589-601.

20. Trudeau F, Shephard RJ. Physical education, school physical activity, school sports and academic achievement. Int J Behav Nutr Phys Act. 2008,5(10):112.

21. Lee DH, Kang S, Zum S. A qualitative assessment of personal and academic stressors among Korean Collage students: an exploratory study. Coll Stud J. 2005;39(3):442-8. 
22. Guedes DP, Lopes CC, Guedes JERP. Reprodutibilidade e validade do Questionário Internacional de Atividade Física em adolescentes. Rev Bras Med Esporte. 2005;11(2):151-64.
23. Hulley SB, Cummings SR, Browner WS, Grady DG. Delineando a pesquisa clínica - uma abordagem epidemiológica. $3^{\mathrm{a}}$ ed. Porto Alegre: Artmed; 2008.

\section{Endereço para correspondência:}

Ana Maria Fontenelle Catrib

Universidade de Fortaleza

Programa de Pós-Graduação em Saúde Coletiva

Av. Washington Soares, 1321

Bairro: Edson Queiroz

CEP. 60811-905 - Fortaleza - CE - Brasil

E-mail: catrib@unifor.br 


\section{INSTRUMENTO DE AVALIAÇÃO DA PROMOÇÃO DA SAÚDE NA UNIVERSIDADE (IAPSU)}

Estimado(a) estudante, esta pesquisa busca conhecer sua percepção sobre a promoção da saúde na universidade e, por meio de sua opinião, será possível compreender os elementos que ajudam a construir um ambiente universitário saudável. Suas respostas são absolutamente confidenciais e pedimos para exprimi-las com toda sinceridade.

\section{Parte 1}

Dados de identificação

\begin{tabular}{|c|c|c|c|c|c|}
\hline Sexo & $\square$ Feminino & $\square$ Masculino & & & \\
\hline Raça ou cor & $\square$ Branco & $\square$ Negro & $\square$ Mulato/Pardo & $\square$ Amarelo & $\square$ Índio \\
\hline Estado civil & $\square$ Solteiro(a) & $\square$ Casado(a) & $\square$ União Consensual & $\square$ Viúvo(a) & $\square$ Separado(a) \\
\hline Religião/Crença & $\begin{array}{l}\square \text { Católica } \\
\square \text { Outra }\end{array}$ & $\begin{array}{l}\square \text { Protestante } \\
\square \text { Nenhuma }\end{array}$ & $\square$ Espírita & $\square$ Judaica & $\square$ Islâmica \\
\hline Renda Familiar & $\begin{array}{l}\square<1 \mathrm{SM} \\
\square 11-14 \mathrm{SM}\end{array}$ & $\begin{array}{l}\square 1 \mathrm{SM} \\
\square 14-17 \mathrm{SM} \\
\end{array}$ & $\begin{array}{l}2 \text { a } 5 \text { SM } \\
\square 17-20 \text { SM }\end{array}$ & $\begin{array}{l}\square 5-8 \mathrm{SM} \\
\square+20 \mathrm{SM}\end{array}$ & $\begin{array}{l}\square 8-11 \mathrm{SM} \\
\square \mathrm{NS}\end{array}$ \\
\hline
\end{tabular}

SM=Salário mínimo; NS=Não sei

\section{Dados referentes aos pais}

\begin{tabular}{|c|c|c|c|c|c|c|c|c|}
\hline \multicolumn{2}{|c|}{$\begin{array}{c}\text { Superior } \\
\text { Completo }\end{array}$} & \multicolumn{2}{|c|}{$\begin{array}{l}\text { Superior } \\
\text { Incompleto }\end{array}$} & $\begin{array}{l}\text { Médio } \\
\text { Completo }\end{array}$ & $\begin{array}{l}\text { Médio } \\
\text { Incompleto }\end{array}$ & $\begin{array}{l}\text { Fundamental } \\
\text { Completo }\end{array}$ & $\begin{array}{l}\text { Fundamental } \\
\text { Incompleto }\end{array}$ & Não alfabetizada(o) \\
\hline Pai & $\square$ & & & $\square$ & $\square$ & $\square$ & $\square$ & $\square$ \\
\hline Mãe & $\square$ & & & $\square$ & $\square$ & $\square$ & $\bar{\square}$ & $\square$ \\
\hline \multicolumn{3}{|c|}{ Você trabalha atualmente? } & \multicolumn{2}{|c|}{$\square \operatorname{Sim}$} & $\square$ Não & & & \\
\hline \multicolumn{2}{|c|}{ Se sim, } & \multicolumn{3}{|c|}{$\begin{array}{l}\square 10 \text { a } 20 \\
\mathrm{~h} / \text { semanais }\end{array}$} & $\begin{array}{l}\mathrm{21} \text { a } 30 \\
\mathrm{~h} / \text { semanais }\end{array}$ & $\begin{array}{l}\square 31 \text { a } 40 \\
\mathrm{~h} / \text { semanais }\end{array}$ & $\begin{array}{l}\square \text { acima de } 40 \\
\text { h/ semanais }\end{array}$ & \\
\hline
\end{tabular}

\section{Parte 2}

\section{Domínio 1- Atividade física}

1. Você participa de atividades físicas pro- $\quad \square$ Sim $\quad \square$ Não movidas pela universidade?
2. Se sim, qual atividade?
$\square$ Musculação
$\square$ Natação
$\neg$ Futebol
$\square$ Atletismo
Handebol $\square$ Tênis
$\square$ Voleibol
$\square$ Basquete
$\square$ Outra

3. Tem participado de atividades físicas recreativas na $\quad \square$ Sim $\quad \square$ Não universidade?
4. Se sim,

\begin{tabular}{|c|c|}
\hline$\square$ Gincanas & $\square$ Ginástica \\
\hline$\square$ Corridas & $\square$ Outras \\
\hline
\end{tabular}




\begin{tabular}{|c|c|c|c|c|c|}
\hline $\begin{array}{l}\text { 5. Para você, os espaços da universidade } \\
\text { destinados a atividades físicas são? }\end{array}$ & (1) Muito Ruim & (2) Ruim & (3) Regular & (4) Bom & (5) Muito Bom \\
\hline
\end{tabular}

\section{A Universidade}

(Marque todas as opções presentes na sua universidade)

$\operatorname{Sim}$

Não

Oferece espaço adequado para a prática de exercício físico

Oferece local de banho e troca de roupa

Disponibiliza bebedouro

Possui espaço para guardar bicicletas

Possui academia de ginástica

Possui quadra de esporte

Possui piscina

Possui pista de corrida

Possui campo de futebol

Outros:

7. Para você a prática de atividade física influencia na sua saúde e bem-estar? $\square$ Sim

$\square$ Não

\begin{tabular}{llll} 
8. Por quê? & $\begin{array}{l}\text { Sinto mais disposição } \\
\text { para as atividades diárias }\end{array} \quad \begin{array}{l}\text { Melhora a minha } \\
\text { integração social }\end{array} \quad \begin{array}{l}\text { Melhora o meu } \\
\text { bem-estar emocional baz com que eu me sinta } \\
\text { Outros: }\end{array}$ \\
\hline
\end{tabular}

9. Os membros da sua família participam de atividade física e/ou recreativa?

\begin{tabular}{lcc} 
Pai & $\square \operatorname{Sim}$ & $\square$ Não \\
\hline Mãe & $\square \operatorname{Sim}$ & $\square$ Não \\
\hline Irmãos & $\square \operatorname{Sim}$ & $\square$ Não \\
\hline
\end{tabular}

\section{Domínio 2 - Alimentação}

10. Os restaurantes e/ou lanchonetes da universidade oferecem alimentos saudáveis (pobres em gordura, ricos em fibra, vitaminas, minerais, proteínas e calorias)?

11. Marque as opções que você considera ser alimentação saudável.

Fazer no mínimo três alimentações ao dia

Fazer esforço para comer alimentos sem gordura

Incluir fibras na alimentação

Beber pelo menos 1.5 litros de água por dia

Incluir proteínas, calorias, vitaminas e minerais nas refeições

Outros:

12. Para você, a universidade oferece ambiente confortável para fazer sua alimentação?

$\operatorname{Sim}$

$\square$ Não

13. Por quê?

O ambiente é limpo

$\mathrm{O}$ ambiente é tranquilo

O ambiente tem clima agradável

O ambiente é silencioso

O ambiente é acolhedor

Outros: 


\section{Domínio 3 - Fatores ambientais}

16. O uso consciente de água e energia é tratado em sua universidade? $\square \operatorname{Sim}$ $\square$ Não

17. Em sua universidade as instalações sanitárias estão limpas e em funcionamento? $\square \operatorname{Sim}$ Não

18. Existem lixeiras com tampa nas salas de aula, pátios, banheiros e corredores? $\square \operatorname{Sim}$ $\square$ Não

19. As salas de aula são adequadas em relação a: Sim Não

\begin{tabular}{lcc}
\hline Iluminação & $\square$ & $\square$ \\
\hline Ventilação & $\square$ & $\square$ \\
\hline Acústica & $\square$ & $\square$ \\
\hline Mobiliário & $\square$ & $\square$ \\
\hline
\end{tabular}

20.Você se sente seguro(a) em sua (1) Nada seguro (2) Pouco seguro (3) Razoavelmente seguro universidade?

Domínio 4 - Fatores psicossociais e consumo de álcool e drogas

21.Você tem participado de atividades relacionadas aos temas abaixo? Atividades que promovem a convivência universitária

Atividades que abordam a autoestima e o autoconhecimento

Atividades reflexivas sobre o planejamento de vida Atividades que incentivam comportamentos promotores de saúde Atividades que promovem a participação social

Atividades sobre cidadania

Atividades sobre sexualidade

Atividades sobre afetividade e relacionamentos interpessoais

Atividades culturais (dança, teatro, pintura, folclore, outras)

Atividades recreativas e lazer

Outras

22. De quais atividades acadêmicas você participa?

Sim Não

Centro acadêmico

\begin{tabular}{lcc}
\hline Extensão universitária & $\square$ & $\square$ \\
\hline Monitoria & $\square$ \\
\hline Programas de iniciação científica & $\square$ & $\square$ \\
\hline Atividades ecológicas & $\square$ & $\square$ \\
\hline Atividades sobre cidadania & $\square$ & $\square$ \\
\hline Outras & \\
\hline
\end{tabular}

23. Em geral, como você se sente na

(1) Muito mal

(2) Mal

(3) Regular

(4) Bem

(5) Muito Bem universidade? 
24. Em geral, como os alunos se tratam na

(1) Muito mal

(2) Mal

(3) Regular

(4) Bem

(5) Muito Bem universidade?

25. Em geral, como os alunos e os (1) Muito mal

(2) Mal

(3) Regular

(4) Bem

(5) Muito Bem professores se relacionam?

26. Em geral, como é a relação entre os

(1) Muito mal

(2) Mal

(3) Regular

(4) Bem

(5) Muito Bem

alunos e a coordenação do curso?

27. Existem situações de violência na universidade?

$\square \operatorname{Sim}$

Não

28. Se sim,

$\operatorname{Sim}$

Não

Violência física

Violência psicológica

Violência de gênero

Violência sexual

29. Se você respondeu sim na questão 32, responda.

Quem pratica a violência?

Alunos

Professores

Funcionários

Pessoas externas à instituição

30. Em geral, como são tratados os estudantes

(1) Muito mal

(2) Mal

(3) Regular

(4) Bem

(5) Muito Bem

obesos na universidade?

31. Em geral, como são tratados os estudantes

(1) Muito mal

(2) Mal

(3) Regular

(4) Bem

(5) Muito Bem

homoafetivos na universidade?

32. Em geral, como são tratados os estudantes

(1) Muito mal

(2) $\mathrm{Mal}$

(3) Regular

(4) Bem

(5) Muito Bem com deficiência física na universidade?

33. O que é uma universidade saudável para você?

Sim

Não

Instituição que cuida da saúde e do bem-estar dos estudantes, professores, funcionários e da comunidade do seu entorno

Instituição que oferece orientações e campanhas que incentivam um estilo de vida promotor de saúde

Instituição que mantém parcerias com serviços de saúde para os estudantes, funcionários e a

comunidade do seu entorno

Instituição que proporciona interações sociais (festas culturais, calouradas, saraus)

Instituição que disponibiliza espaço adequado para a realização das atividades físicas e condições para uma alimentação saudável

Instituição que possui campus arborizado e bem cuidado

Instituição que oferece orientações e palestras sobre administração do estresse

Instituição que envolve os alunos, professores e comunidade nas suas decisões estratégicas 
Outras drogas:

36. Seus familiares consomem álcool, tabaco, medicamento ou alguma droga ilícita?

37. Se sim, assinale qual(is).

Membro

Álcool

Tabaco

Medicamento

Droga Ilícita

Pai
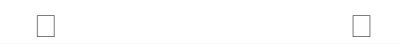

$\square$
$\square$
$\square$

(20)

38. Você tem participado de atividades de prevenção de consumo de álcool e droga na universidade?

$\square$ Sim $\square$ Não

Domínio 5 - Práticas Integrativas e Complementares

39. Você sabe o que são as Práticas Integrativas e Complementares?

$\square \operatorname{Sim}$

$\square$ Não

40. Você acha que Prática Integrativa e Complementar na universidade pode promover ambiente saudável?

$\square \operatorname{Sim}$

41. Você sabe o que é e já usou alguma das práticas do quadro abaixo:

Prática

Você sabe o que é?

Usa ou já usou?

\begin{tabular}{|c|c|c|c|c|}
\hline & Sim & Não & Sim & Não \\
\hline Acupuntura & $\square$ & $\square$ & $\square$ & $\square$ \\
\hline Aromaterapia & $\square$ & $\square$ & $\square$ & $\square$ \\
\hline Benzeção & $\square$ & $\square$ & $\square$ & $\square$ \\
\hline Cristais & $\square$ & $\square$ & $\square$ & $\square$ \\
\hline Cromoterapia & $\square$ & $\square$ & $\square$ & $\square$ \\
\hline Dieta Qual? & $\square$ & $\square$ & $\square$ & $\square$ \\
\hline Ervas Medicinais & $\square$ & $\square$ & $\square$ & $\square$ \\
\hline Florais & $\square$ & $\square$ & $\square$ & $\square$ \\
\hline Hidroterapia & $\square$ & $\square$ & $\square$ & $\square$ \\
\hline Homeopatia & $\square$ & $\square$ & $\square$ & $\square$ \\
\hline Iridologia & $\square$ & $\square$ & $\square$ & $\square$ \\
\hline Lian Gong & $\square$ & $\square$ & $\square$ & $\square$ \\
\hline Massagem & $\square$ & $\square$ & $\square$ & $\square$ \\
\hline Meditação & $\square$ & $\square$ & $\square$ & $\square$ \\
\hline Moxabustão & $\square$ & $\square$ & $\square$ & $\square$ \\
\hline Musicoterapia & $\square$ & $\square$ & $\square$ & $\square$ \\
\hline Ortomolecular & $\square$ & $\square$ & $\square$ & $\square$ \\
\hline Quiropatia & $\square$ & $\square$ & $\square$ & $\square$ \\
\hline Reflexologia & $\square$ & $\square$ & $\square$ & $\square$ \\
\hline Reiki & $\square$ & $\square$ & $\square$ & $\bar{\square}$ \\
\hline Relaxamento & $\square$ & $\square$ & $\square$ & $\square$ \\
\hline Outro Qual? & $\square$ & $\square$ & $\square$ & $\square$ \\
\hline
\end{tabular}

Obrigado por responder ao questionário. Antes de finalizar, verifique se você respondeu a todas as perguntas. 